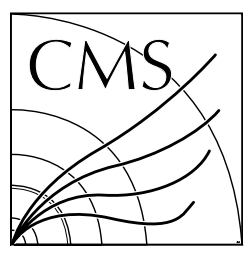

The Compact Muon Solenoid Experiment

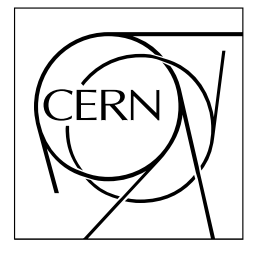

08/09/2000

\title{
Study of radiation damage and substrate resistivity effects from beam test of silicon microstrip detectors using LHC readout electronics.
}

M. M. Angarano, M. Biasini, G.M. Bilei, M.T. Brunetti, B. Checcucci, D. Creanza, M. de Palma, L. Fanò, L. Fiore, M. Giorgi, P. Lariccia, G. Maggi, G. Mantovani, G. Messina, S. My, A. Papi, V. Radicci, R. Santinelli, G. Selvaggi, L. Servoli, L. Silvestris, P. Tempesta

INFN and Dip. Interateneo di Fisica, Bari, Italy

INFN and University of Perugia, Perugia, Italy

\begin{abstract}
We present beam test results on single sided silicon microstrip detectors, with different substrate resistivities. The effects of radiation damage are studied on a detector irradiated at a fluence of $2.4 \cdot 10^{14}$ $n / \mathrm{cm}^{2}$. The detectors are read out with the APV6 chip, which is compatible with the $40 \mathrm{MHz}$ LHC clock. The performance of different detectors and readout modes are studied in terms of signal-tonoise ratio and efficiency.
\end{abstract}




\section{Introduction}

The tracking system of the CMS experiment at the future LHC collider, based on silicon mictrostrip detectors, will be exposed to ionizing radiation with an equivalent fluence up to about $1.6 \cdot 10^{14} \mathrm{n} / \mathrm{cm}^{2}$ after 10 years of operation[1] at a radial distance of $22 \mathrm{~cm}$ from the interaction point. This irradiation will affect the most important detector parameters, which conversely will give a degradation of detector performances, namely the noise level and the charge collection. Previous studies on electrical performance of microstrip detectors[2] have indicated the advantages of using substrates with low resistivity $(1-4 \mathrm{~K} \Omega \cdot \mathrm{cm})$, related to the possibility of mantaining a relatively low bias voltage after the type-inversion due to radiation damage.

In this paper we investigate the performances on muon beam of $p^{+}$on $n$-type detectors with different substrate resistivity, before and after irradiation. In addition, the APV6 chip is used as 25ns front-end electronic, and a comparison between the two foreseen readout modes is presented. The attention is focused on signal-to-noise ratio and efficiency for the different resistivities, irradiation and readout modes.

\section{Detectors description}

The detectors here studied are $<111>$ single sided, AC coupled, with $p^{+}$strips $18 \mu$ m width, implanted over an $n$ type substrate, produced by CSEM. The active area of the detector is $57.4 \times 57.4 \mathrm{~mm}^{2}$, the thickness is about $300 \mu \mathrm{m}$. The total number of strips is 1024 and the pitch is $61 \mu \mathrm{m}$. Two types of detectors with different resistivity are used: $1-2 \mathrm{k} \Omega \cdot \mathrm{cm}$ (Low Resistivity) and $4-8 \mathrm{k} \Omega \cdot \mathrm{cm}$ (High Resistivity). The bias is obtained through $2 \mathrm{M} \Omega$ polysilicon resistors.

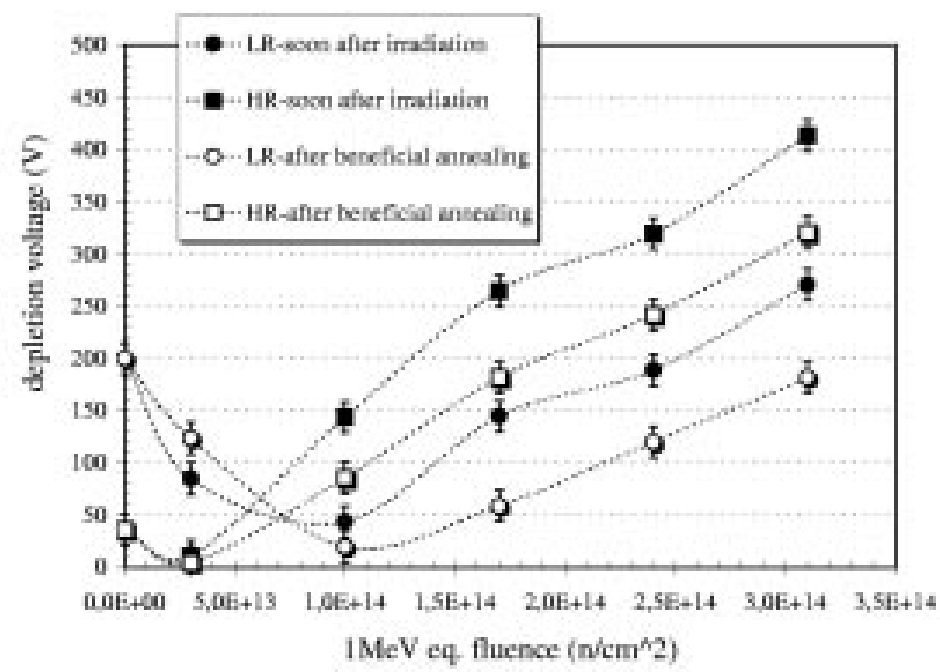

Figure 1: Depletion voltage as a function of fluence: LR vs. HR comparison.

Before irradiation, a low resistivity device has a relatively high value of effective doping concentration $\left(N_{e f f}\right)$, and therefore a high depletion voltage $\left(V_{d e p} \propto N_{e f f}\right)$. The inversion fluences $\phi_{i n v}$ (defined as the fluences at which an $n$-type silicon becomes "effectively" $p$-type) depends on $N_{\text {eff }}$ as this empirical formula shows:

$$
\phi \simeq(17 \div 20) N_{\text {eff }}
$$

where $\phi_{i n v}$ is expressed in $\mathrm{cm}^{-2}$ and $N_{\text {eff }}$ in $\mathrm{cm}^{-3}$. As shown in figure $1[2]^{1)}$, the low resistivity device compared to the high resistivity one, has the type inversion at larger fluences and the depletion voltage, after the type inversion, lower than one hundred Volts at the same fluence.

Two detectors are bonded together to form a single readout module of about $12 \mathrm{~cm}$ length equipped with 2 APV6 chips in the central zone to readout 256 strips. Three modules have been assembled: one with high resistivity substrate (HR-NI), one with low resistivity substrate (LR-NI) and a last one (LR-IR), low resistivity, irradiated with a dose of $2.4 \times 10^{14} \mathrm{n} / \cdot \mathrm{cm}^{2}$. Irradiation was performed at the ENEA facility in Casaccia (Roma), at room temperature and without biasing the detectors. To avoid anti-annealing effects, the detectors, after irradiation, are kept at a temperature $\simeq-10^{\circ} \mathrm{C}$.

1) The first set of measures was performed just after irradiation, the second one, after benefical annealing, 15-days after. 


\section{APV6 Description}

The APV6 chip[4] is equipped with 128 analog channels, each one containing a pipeline memory with 160 locations. The two pointers for read-write operation are driven by the $40 \mathrm{MHz}$ LHC clock, and are separated by a time interval called latency.

There are two different operation modes for the APV6: peak and deconvolution. In the first case a full sampling of the signal is done, and the fall time for the signal reconstruction is $100 \mathrm{~ns}$; in deconvolution mode only 3 samples are needed and the fall time is reduced to $25 \mathrm{~ns}$. The introduction of a deconvolution filter gives an increase of about $40 \%$ for the electronic noise and a charge loss of about 20\%[5].

The APV6 response can be fine tuned using different programmable registers as shown in table 1. An example of this kind of studies will be shown in section 6 .

Table 1: Internal APV6 registers

\begin{tabular}{|c|c|c|c|c|}
\hline Register & Class & Range & Resolution & Default \\
\hline \hline IPRE & $I$ & $0-1020 \mu A$ & $4 \mu A$ & $440 \mu A$ \\
$I S H A$ & $I$ & $0-255 \mu A$ & $1 \mu A$ & $88 \mu A$ \\
$I P S P$ & $I$ & $0-127.5 \mu A$ & $0.5 \mu A$ & $42 \mu A$ \\
$V P R E$ & $V$ & $V D D-V S S$ & $18 m V$ & $-0.7 V$ \\
$V S H A$ & $V$ & $V D D-V S S$ & $18 m V$ & $2.0 V$ \\
$V A D J$ & $V$ & $G N D-V S S$ & $9 m V$ & $200 \mu A$ \\
$V C A S$ & $V$ & $G N D-1.02 V$ & $4 m V$ & $0 V$ \\
$C L V L$ & $Q$ & $0-15.3 \mathrm{fC}$ & $0.06 \mathrm{fC}$ & Off \\
\hline
\end{tabular}

IPRE

IPSP

VSHA

VCAS
Preamplifier bias current APSP bias current Shaper feedback bias voltage Cascade voltage bias level
ISHA

VPRE

VADJ

CLVL
Shaper bias current

Preamplifier feedback bias voltage

Output level adjustment

Reference for internal test system

\section{Experimental setup}

The modules are placed inside a thermally insulated box in wich nitrogen is flushed. The temperature is set at $-10^{\circ} \mathrm{C}$ and monitored to be stable within $\pm 1^{\circ} \mathrm{C}$. The modules are readout by a VME-based DAQ system [6]. The detectors are biased in a range up to about 3 times the depletion voltage. The measured depletion voltage is $V_{d}=40$, 150, 200 Volts for HR-NI, LR-IR and LR-NI.

The modules are tested using a $100 \mathrm{GeV}$ muon beam at X5 CERN area. The trigger is defined by the coincidence of 2 scintillators placed in front of the detectors. The active area of the 2 scintillators is $2 \times 2 \mathrm{~cm}^{2}$ in the first part of the data taking and $6 \times 6 \mathrm{~cm}^{2}$ in the second one. No precise tracking is performed during the acquisition due to the absence of the telescope system. Several million triggers were collected during about 10 days of experiment.

\section{Analysis method}

The data analysis is performed using the ORCA package, developed at CERN by the CMS collaboration [7]. The strip signal $\left(\mathrm{S}_{i}\right)$ is calculated from the raw digital value subtracting the strip pedestal and the common mode fluctuation. The strip noise $\left(\mathrm{N}_{i}\right)$ is calculated as the statistical fluctuation of the strip signal, excluding the hits caused by a particle. When strip signal and noise are defined the program try to find a cluster. The strips for which the ratio $S_{i} / \mathrm{N}_{i}$ is greater than a threshold $\mathrm{T}_{s}$ are identified as seed of a possible cluster. The neighbouring strips are associated to the cluster if their ratio $\mathrm{S}_{i} / \mathrm{N}_{i}$ is greater than $\mathrm{T}_{n}$. For $N$ strips selected in this way, the cluster signal $\left(\mathrm{S}_{c l}\right)$ and cluster noise $\left(\mathrm{N}_{c l}\right)$ are calculated as:

$$
\mathrm{S}_{c l}=\sum_{i=1}^{N} S_{i} \quad \mathrm{~N}_{c l}=\frac{S_{c l}}{\sum_{i=1}^{N} \frac{S_{i}}{N_{i}}}
$$

Finally, if the signal-to-noise ratio $S_{c l} / N_{c l}$ exceeds the value $\mathrm{T}_{c}$, the cluster is considered for further analysis. The values used for the cluster finding thresholds are: $\mathrm{T}_{s}=4, \mathrm{~T}_{n}=2$ and $\mathrm{T}_{t}=5$. 

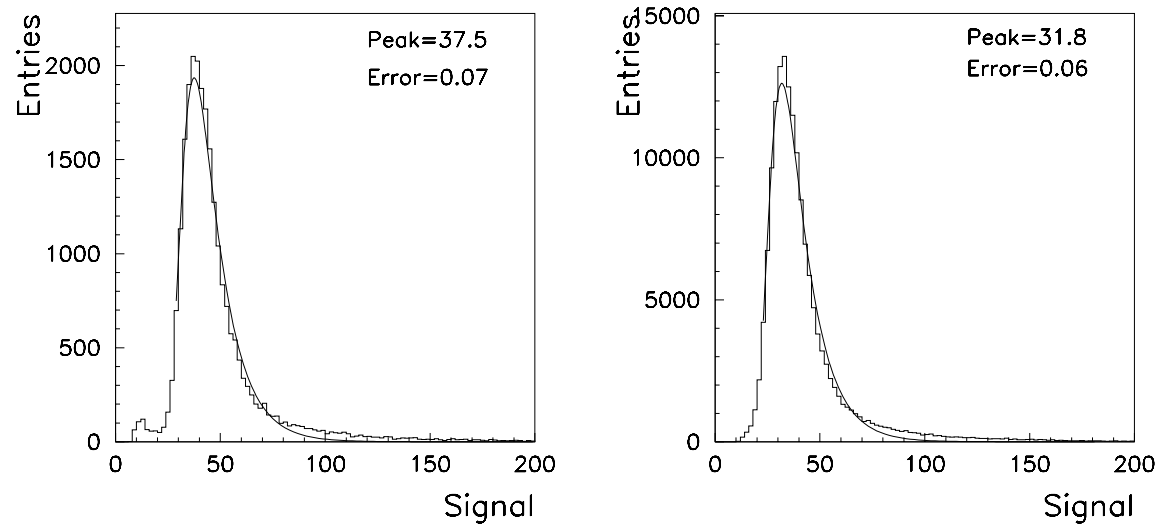

Figure 2: Cluster signal in peak (left) and deconvolution mode (right)
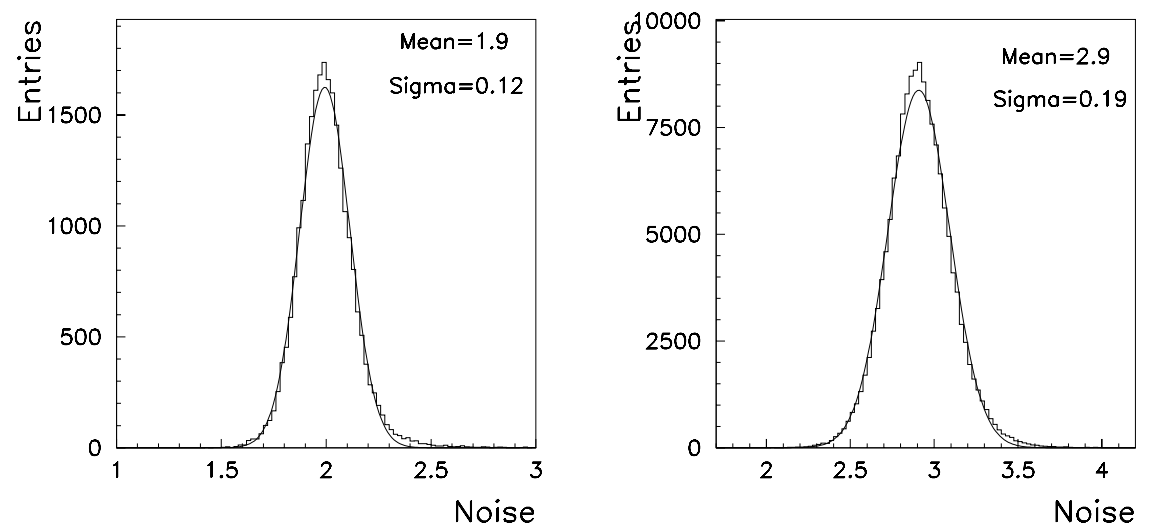

Figure 3: Cluster noise in peak (left) and deconvolution mode (right)

The most probable value of the Landau function which best fits the $S_{c l}$ distribution defines the detector signal, while the most probable value of the Gaussian function fitting the $N_{c l}$ distribution defines the detector noise (figures 2 and 3 ).

\section{Experimental results}

\subsection{APV6 parameters}

The dependence of the detectors performance on the APV6 parameters is studied through a variation of the latency and of the shaper feedback bias voltage, VSHA. For the other parameters of the chip the defaults settings are used.

Fig. 4 a) shows the $\mathrm{S} / \mathrm{N}$ for the high resistivity not irradiated detector (HR-NI) versus the latency value, for both APV6 operational modes: peak and deconvolution. The two signal-to-noise maximum values are separated in time by 3 latency units (1 latency unit $=25 \mathrm{~ns}$ ) plus 8ns, as expected [8]. The low-resistivity, irradiated and not irradiated (LR-IR and LR-NI) detectors, exhibit the same time difference. For deconvolution mode, the width of the distribution is narrower with respect to the one for peak mode, for all three kinds of detectors (e.g. figure 4 for the HR-NI detector). This behaviour confirms that the signal sampling time is particularly important for the charge collection optimization in deconvolution mode.

From the study done by varying the parameter VSHA for the HR-NI detector, an increase of the cluster signal is found for increasing values of VSHA (see figure 5). The VSHA parameter seems have no effect on the cluster noise. The percentage increase of the signal, from the minimum to the maximum value of VSHA, is about $45 \%$ in peak mode and $14 \%$ in deconvolution mode. 


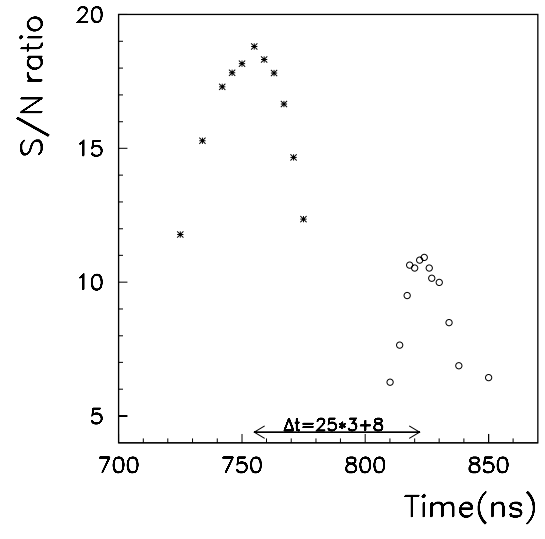

(a)

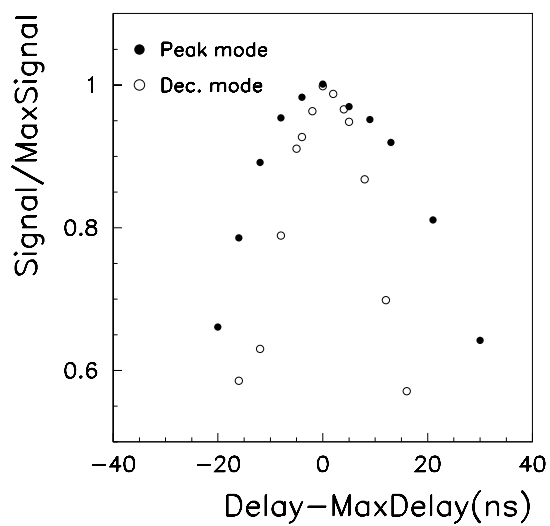

(b)

Figure 4: Signal-to-noise ratio as a function of the latency (a) in peak and deconvolution mode (full and empty circles, respectively) for the HR-NI detector. Relative signal for the HR-NI detector as a function of latency subtracted delay (b) for both readout modes.

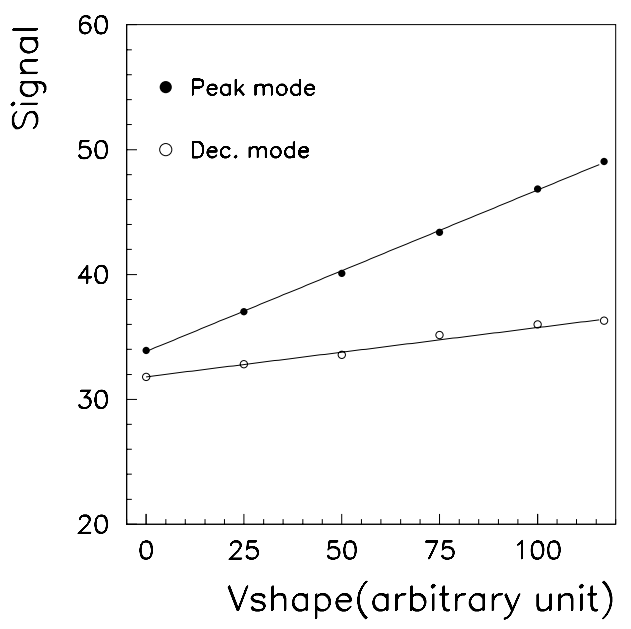

Figure 5: Cluster signal as a function of the VSHA register for the HR-NI detector, in peak and deconvolution mode (black and white circles, respectively)

\subsection{Cluster noise and signal-to-noise ratio}

A simple theoretical model is used to estimate the signal-to-noise value taking into account the main noise sources in a silicon detector coupled with the front-end electronics: the amplifier noise, the shot noise of the strip leakage current $I_{s}$, the thermal noise due to the bias resistor $R_{p o l}$ and metal resistance $R_{s}$ [9]. The analytical formulas of each component are reported in table 2. The total noise, expressed in terms of an equivalent noise charge (ENC), is the sum in quadrature of the four noise sources. Not all the released charge is readout by the electronics because of the AC coupling capacitor. The signal-to-noise ratio can be calculated assuming the injected fractional charge equal to $22400 e^{-}$.

For the noise calculation, we use the values of resistance and leakage current measured in laboratory. $C_{t o t}$ can be calculated from:

$$
\frac{1}{C_{t o t}}=\frac{1}{C_{a c}}+\frac{1}{C_{b a c k}+2\left(C_{i i}+C_{i i+1}\right)}
$$

where $C_{a c}$ is the coupling capacitance, $C_{i i}$ is the interstrip capacitance, $C_{i i+1}$ is the second neighbour interstrip 


\begin{tabular}{|l|l|l|l|}
\hline Noise source & Type & Expression at $\mathrm{t}=-10 \mathrm{C}$ & Deconvolution \\
\hline Strip leakage current $I_{b}$ & Parallel & $\simeq 108 \sqrt{\tau(n s) I_{s}(\mu A) e^{-}}$ & $\mathrm{x} 0.45$ \\
\hline Polarization resistors $R_{p}$ & Parallel & $\simeq 22.5 \sqrt{\frac{\tau(n s)}{R_{p o l}(M \Omega)}} e^{-}$ & $\mathrm{x} 0.45$ \\
\hline Metal strip resistance $R_{s}$ & Series & $\simeq 13 C_{\text {tot }} \sqrt{\frac{R_{s}(\Omega)}{\tau(n s)} e^{-}}$ & $\mathrm{x} 1.45$ \\
\hline Front-end electronics & Series & $510+36 C_{\text {tot }}$ & $1000+46 C_{\text {tot }}$ \\
\hline
\end{tabular}

Table 2: Noise sources. $C_{t o t}$ is the total capacitive load seen from the amplifier input into the detector, $\tau$ is the shaping time.

capacitance and $C_{\text {back }}$ is the backplane capacitance. Before irradiation we can assume that the $C_{\text {tot }} \simeq 13 p F$ for the high and low resistivity detector; after irradiation $C_{t o t} \simeq 19 p F$ at $V_{d e p}$ and $C_{t o t} \simeq 17 p F$ at $V_{\text {bias }} \sim 2 V_{\text {dep }}$. As expected from the APV characteristics, the noise is higher in deconvolution than in peak mode. In table 3 , we compare the calculated and the measured signal-to-noise ratio. Even if the model used is very simple, we obtain a substantial agreement between measurements and calculations in each configuration.

\begin{tabular}{|c|c|c|c|c|}
\hline & \multicolumn{2}{|c|}{ Before irradiation } & \multicolumn{2}{c|}{ After irradiation } \\
\hline & peak & deconvolution & peak & deconvolution \\
\hline Expected & 21.4 & 13.4 & 14. & 11.6 \\
\hline Measured & 18.7 & 12. & 13. & 8.7 \\
\hline
\end{tabular}

Table 3: Comparison between the estimated and measured signal-to-noise ratios for the low resistivity detectors.

\section{3 $\eta$-function}

In order to check the charge sharing between adjacent strips, the charge of the two strips in the cluster with highest signal is studied. Figure 6 shows the distribution of the response function $\eta=Q_{l} /\left(Q_{l}+Q_{r}\right)$, where $Q_{l}$ and $Q_{r}$ are the charges collected by the two strips, according to readout order. The distributions for clusters with one, two and three strips are compared, both for peak and deconvolution mode. In all cases the distributions are nearly symmetric, as expected.
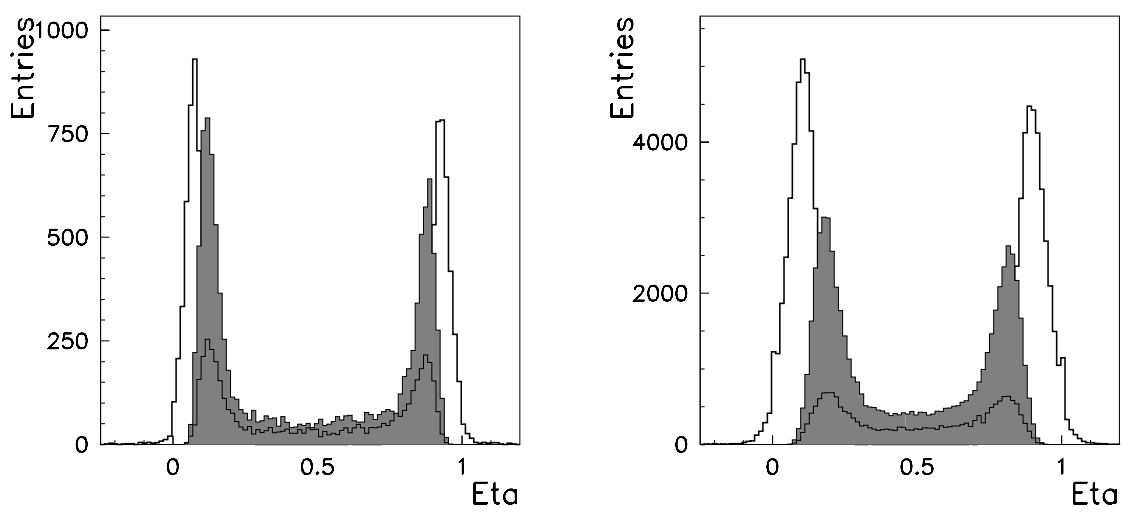

Figure 6: Distribution of the response function $\eta$ for the high resistivity detector in peak (left) and deconvolution mode (right); black $=1$ strip cluster, dashed $=2$ strip clusters, dark $=3$ strip clusters

\subsection{Bias scan}

The behavior of the detectors is tested for different values of the bias voltage. This scan is done for all detectors in peak mode and for low-resistivity detectors in deconvolution mode too. We have studied the dependence on bias voltage of signal, noise, signal-to-noise ratio, mean cluster width and detection efficiency.

We have verified that the low resistivity detectors can operate up to $500 \mathrm{~V}$ [1] before and after irradiation. As shown in fig.7, the cluster noise does not depend on applied voltage, as expected. The dependence of cluster signal 

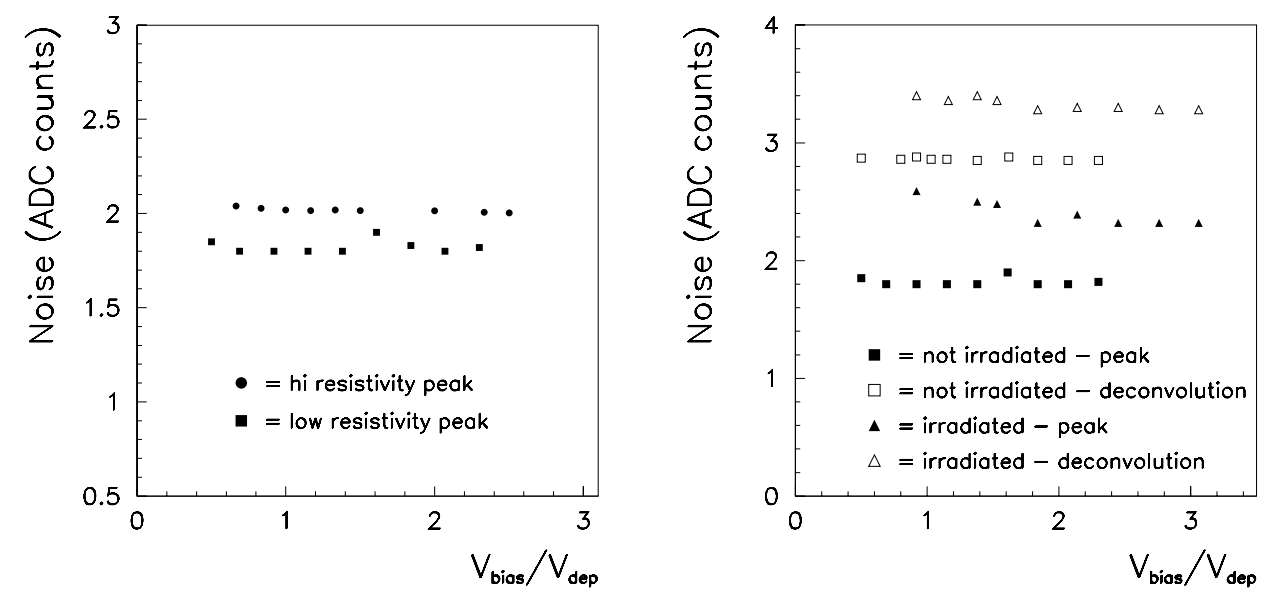

Figure 7: - Left - Cluster noise vs $V_{\text {bias }} / V_{\text {dep }}$ for HR-NI and LR-NI in peak mode - Right - Cluster noise vs $V_{\text {bias }} / V_{\text {dep }}$ for LR-NI, LR-IR in peak and deconvolution mode

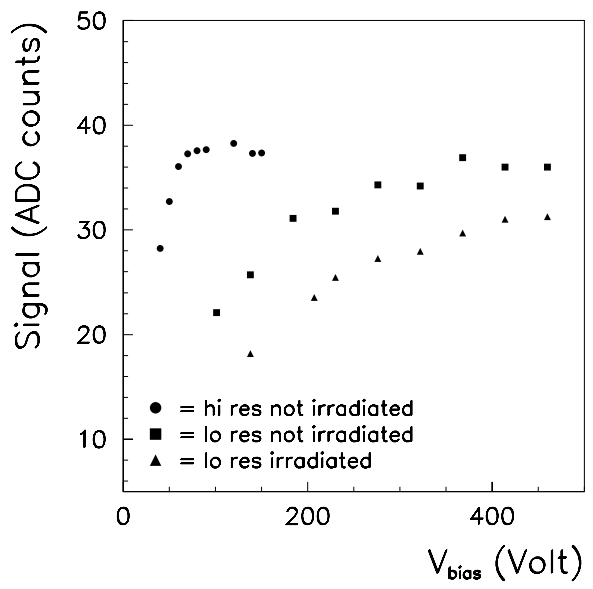

Figure 8: Cluster signal in peak mode as a function of bias voltage for HR-NI, LR-NI and LR-IR modules

on the bias voltage is shown in figure 8. The signal increases with increasing bias voltage, up to a plateau value reached at $\simeq 1.5 V_{\text {dep }}$. The high resistivity detector shows a uniform behavior for large values of the bias scan, therefore it is operated only up to $150 \mathrm{~V}$. The irradiated detector shows a lower collected charge, of about $20 \%$ with respect to not irradiated ones, as expected from radiation damages [1]. Detectors with different resistivities do not present significant differences in performances, as can be inferred from figure 9; the signal-to-noise behavior is very similar for the two detectors, and in agreement with theoretical estimates. In deconvolution mode the detectors have signal-to-noise ratio lower than in peak mode (figure 9) due to the increased noise given by the deconvolution filter [5]. The results obtained in deconvolution mode for the irradiated sensors show the capability of operating the detectors after 15 years of LHC, despite the reduced signal-to-noise ratio with respect to initial performances.

\subsection{Cluster size}

The size of a cluster is mainly determined by the strip pitch, the interstrip capacitance and by the diffusion length of the electrons. Figure 10 shows the mean cluster width for the different detectors studied. The typical dependence of the interstrip capacitance on the bias voltage is reflected here on the cluster width variation as a function of $\left(\mathrm{V}_{\text {bias }}-\mathrm{V}_{\text {dep }}\right) / \mathrm{V}_{\text {dep }}$.

For the irradiated detector there is a larger dependence on the bias voltage due to the larger dependence of the interstrip capacitance on the bias voltage. The variation of the interstrip capacitance, due to radiation damage, 

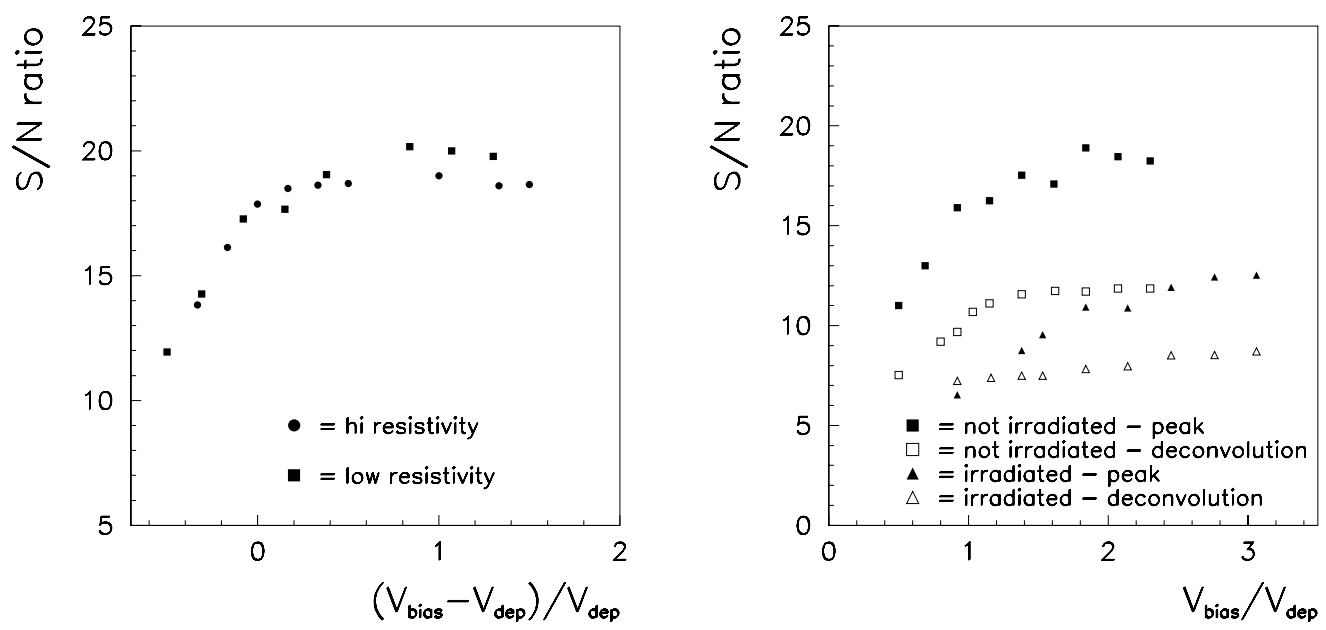

Figure 9: Signal-to-Noise ratio as a function of $\left.\mathrm{V}_{(\text {bias }}-\mathrm{V}_{d e p}\right) / \mathrm{V}_{\text {dep }}$ for the detectors HR-NI, LR-NI in peak mode on the left and for LR-IR, LR-NI in peak and deconvolution mode on the right

gives rise to the increase of the mean cluster width of the irradiated detector, with respect to the non irradiated one with the same resistivity. The mean cluster width for detectors in deconvolution mode is found to be larger than in peak mode; this can be possibly explained by the increase of the noise in deconvolution mode, which gives a larger probability for neighbour strips to be included in the cluster.

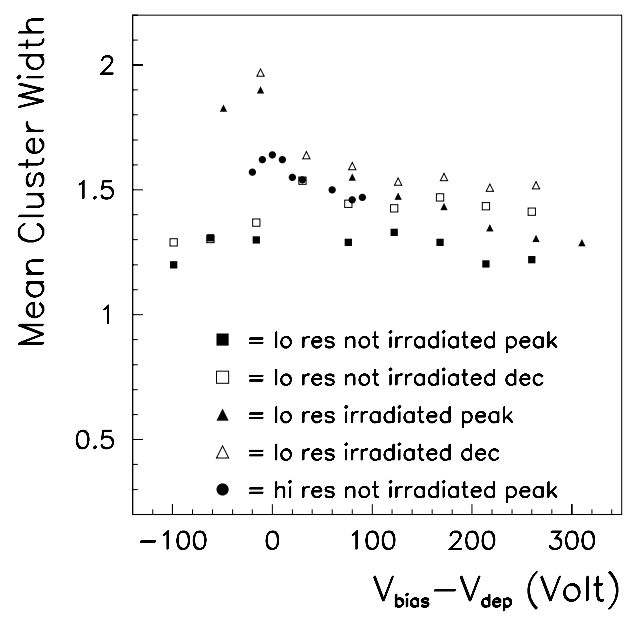

Figure 10: Mean cluster width as a function of $\mathrm{V}_{\text {bias }}-\mathrm{V}_{\text {dep }}$ for the HR-NI (circle), LR-NI (box) and LR-IR (triangle) modules in peak (solid) and deconvolution (empty) mode.

\subsection{Efficiency}

Due to the absence of a high resolution telescope along the beam line, it is not possible to determine the impact position of the tracks on the detectors. Therefore, in order to estimate the efficiency, the same detectors under test are used. The hit efficiency is defined as the ratio between the number of clusters in a detector for which the track extrapolated from the other two detectors matches the hit position ${ }^{2)}$ and the total number of tracks.

A cluster is considered as matched if the hit position is within 3 strips from the predicted position. Figures 11 show the dependence of the efficiency on the signal-to-noise ratio of the clusters and on the latency parameter.

2) The hit position is defined as the charge weighted mean of the strips' position 


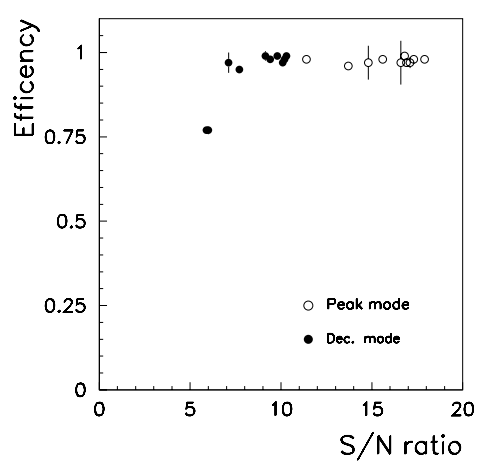

(a)

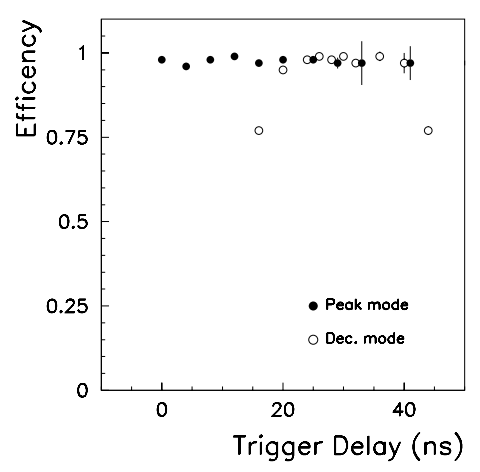

(d)

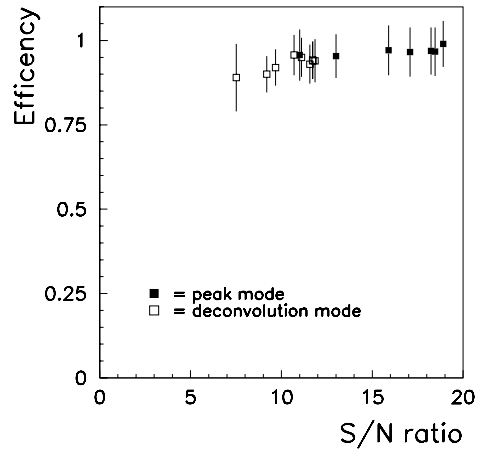

(b)

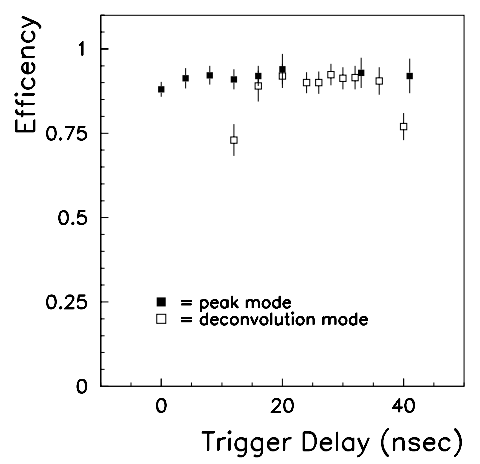

(e)

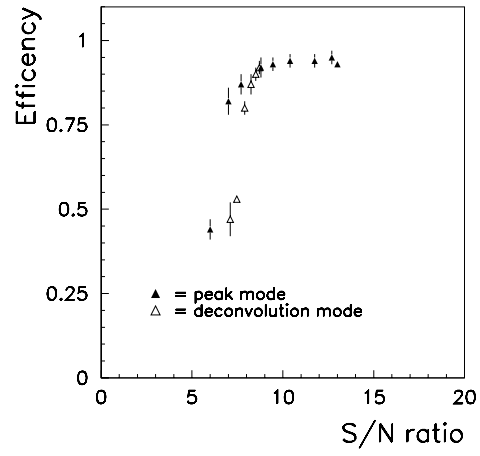

(c)

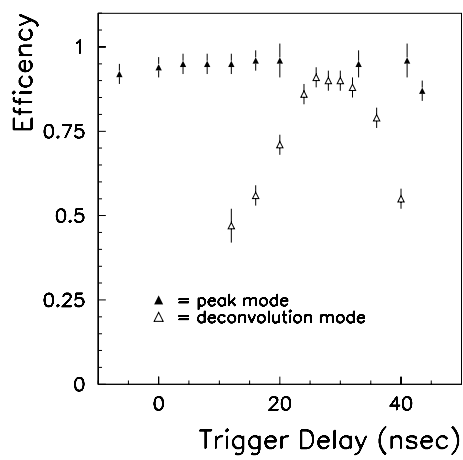

(f)

Figure 11: Efficiency vs S/N in peak and deconvolution mode for: (a) HR-NI, (b) LR-NI and (c) LR-IR. Efficiency vs latency delay in peak and deconvolution for: (d) HR-NI, (e) LR-NI and (f) LR-IR

For not irradiated detectors, both with high and low resistivity, in peak and deconvolution mode, the efficiency is greater than $95 \%$ for signal-to-noise ratios greater than 7 . The irradiated detector reaches efficiencies of about $95 \%$, for signal-to-noise ratios greater than 9. The latency parameter scan (figures $d$, $e$ and $f$ ) shows a substantial independence of the efficiency on this parameter in peak mode, while in deconvolution mode the efficiency is contained within reasonable values only for a narrow window of the latency value. For the irradiated detector this window is considerably reduced, down to about $10 \mathrm{~ns}$.

\section{Conclusions}

The performance on beam test of silicon microstrip detectors, with low $(1-2 \mathrm{k} \Omega \cdot \mathrm{cm})$ and high $(4-8 \mathrm{k} \Omega \cdot \mathrm{cm}) \mathrm{resistiv}$ ity, has been studied. One low resistivity detector was irradiated at a fluence $2.4 \cdot 10^{14} \mathrm{n} / \mathrm{cm}^{2}$. The modules, with $12 \mathrm{~cm}$ strip length and $61 \mu \mathrm{m}$ pitch, have been read-out with the APV6 chip, using both readout modes foreseen, peak and deconvolution. The high and low resistivity detectors before irradiation are found to be very similar in terms of signal-to-noise ratio and detection efficiencies. The low resistivity detectors have been successfully operated up to $500 \mathrm{~V}$ bias voltage. After irradiation, the low resistivity detector maintains a signal-to-noise ratio of about 9 at $400 \mathrm{~V}$ bias, in deconvolution mode, and assures an efficiency greater than $95 \%$. 


\section{References}

[1] CMS, The Tracker Project, Technical Design Report, CERN/LHCC 98-6 CMS TDR (1998).

[2] M.M Angarano, G.M. Bilei, M. Giorgi et al. Characterization of neutron irradiated, low-resistivity silicon detectors, CERN CMS C.R. 2000/06.

[3] D.Passeri, P. Ciampolini, G.M.Bilei A Comprenensive Analysis of Low-Resistivity Silicon Radiation Detectors, CERN CMS C.R. 1999/017.

[4] N. Marinelli, APV logic simulation, CERN CMS NOTE 1999/028.

[5] S. Gadomski, G. Hall et al. The deconvolution method of fast pulse shaping at hadron colliders, NIM A320 (1992) 217-227.

[6] W. Beaumont, Crates Sincronization, CERN web site.

[7] ORCA - website, http://cmsdoc.cern.ch/ORCA/

[8] M. French, APV6 User Manual, Rutherford Appleton Laboratory.

[9] C. Bozzi, Signal-to-Noise evaluations for the CMS silicon microstrip detectors, CERN CMS NOTE 1997/026

[10] M.M. Angarano, A. Bader, D. Creanza, M. de Palma, D. Diacono, S. My, G. Raso, P. Tempesta, Characteristics and SPICE simulation of a single-sided, $n^{+}$on $n$ type Si strip detector before and after neutron irradiation, CERN CMS NOTE 1998/34. 